\title{
BMJ Open Disseminating START: training clinical psychologists and admiral nurses as trainers in a psychosocial intervention for carers of people with dementia's depressive and anxiety symptoms
}

\author{
Kathryn Lord, ${ }^{1}$ Penny Rapaport, ${ }^{2}$ Claudia Cooper, ${ }^{2}$ Gill Livingston ${ }^{2}$
}

To cite: Lord K, Rapaport P, Cooper C, et al. Disseminating START: training clinical psychologists and admiral nurses as trainers in a psychosocial intervention for carers of people with dementia's depressive and anxiety symptoms. BMJ Open 2017;7:e017759. doi:10.1136/ bmjopen-2017-017759

- Prepublication history for this paper is available online. To view these files, please visit the journal online (http://dx.doi. org/10.1136/bmjopen-2017017759).

Received 18 May 2017 Revised 27 June 2017 Accepted 14 July 2017

\section{(a) CrossMark}

${ }^{1}$ Faculty of Health Studies, School of Dementia Studies, University of Bradford, Bradford, UK

${ }^{2}$ Division of Psychiatry, Department of Old Age Psychiatry, University College London, London, UK

Correspondence to Professor Gill Livingston; g.livingston@ucl.ac.uk

\section{ABSTRACT}

Objectives To put into practice and to evaluate an initial dissemination programme for the Strategies for Relatives (START), a clinically and cost-effective manualised intervention for family carers of people with dementia. Setting We offered 3-hour 'train-the-trainer' sessions through the British Psychological Society and Dementia UK. Participants Clinical psychologists and admiral nurses across the UK.

Primary and secondary outcome measures After the training session, attendees completed an evaluation. Attendees were asked how they had implemented START 6 and 12 months later, and to participate in telephone interviews about their experiences of what helps or hinders implementation 1 year after training.

Results We trained 134 clinical psychologists and 39 admiral nurses through 14 training sessions between October 2014 and September 2015 in nine UK locations and made materials available online. The 40 survey respondents had trained 75 other staff. By this time, 136 carers had received START across 11 service areas. Findings from 13 qualitative interviews indicated that some clinical psychologists had begun to implement START, facilitated by buy-in from colleagues, existing skills in delivering this type of intervention, availability of other staff to deliver the intervention and support from the research team. Admiral nurses did not supervise other staff and were unable to cascade the intervention. Where START has not been used, common barriers included lack of staff to deliver the intervention and family carer support not being a service priority. Participants wanted the training to be longer. Conclusions We trained clinical psychologists and admiral nurses to deliver and implement START locally. Results from survey respondents show that it was cascaded further and used in practice in some areas, but we do not know whether START was implemented by nonrespondents. Future dissemination requires management buy-in, availability of practitioners and supervisors and consideration of other ways of delivery.

\section{INTRODUCTION}

The overall economic impact of dementia in the UK alone is $£ 26.3$ billion per year; $44 \%$
Strengths and limitations of this study

- This study adds to the evidence about how to disseminate effective interventions for people with dementia and their families into routine clinical practice.

- The data provide insight into the challenges faced by healthcare professionals working within the National Health Service when trying to implement evidencebased practice.

- We found that while there was enthusiasm to attend dissemination in the form of 'train-the-trainer' for START and the attendees were positive about it, this did not necessarily translate into giving us information about whether other steps were taken, perhaps due to the fact that we were asking them to complete an email survey.

While fidelity to the intervention was emphasised, it was not measured after training.

of this total cost is contributed by the work of unpaid family carers. ${ }^{1}$ As the number of people with dementia increases, policy frameworks and service provision are predicated on the assumption that families will remain the main source of (unpaid) support. ${ }^{1}$ However, family carers are at high risk of psychological morbidity, which increases the likelihood of care home admission of the person with dementia, ${ }^{2}{ }^{3}$ usually against the previous wishes of the person with dementia and with financial consequences for society. ${ }^{1}$

Although in the UK the National Institute for Health and Care Excellence guidelines and the Memory Services National Accreditation Programme quality standards recommend using effective psychosocial interventions for family carers, many carers do not receive them because of costs and lack of staffing or because interventions that do not have an evidence base are embedded in practice. STrAtegies for RelatTives (START) 
is the first manualised intervention for family carers of people living with dementia, delivered by supervised graduate psychologists, demonstrated to be clinically and cost-effective at reducing carer depressive symptoms and improving carer mood over 8 months and 2 years. ${ }^{245}$ Nonetheless, it is not currently widely available in UK clinical services. New interventions often take a long time to be transferred to clinical practice. It is important to find ways to maximise the transition from research evidence to routine care. ${ }^{67}$

We developed and tested START in a randomised controlled trial (RCT), and it fulfilled its primary aims to be clinically effective at reducing dementia carer's anxiety and depressive symptoms in the short term (over 8 months) and the long term (2 years) and to be cost-effective. It also fulfilled secondary aims of improving quality of life and reducing depression in the intervention group. Carers in the treatment as usual group were seven times more likely to have clinically significant depression than those receiving START at 2 years. ${ }^{245}$ It comprises eight manualised sessions delivered to individual carers aimed at supporting them to develop adaptive coping strategies (see box 1 for content of sessions). ${ }^{2}$ Carers were given a manual, including space where they created an individualised plan comprising strategies they intended to continue using and an audio resource with the relaxation

\section{Box 1 Structure of STrAtegies for RelaTives programmes}

\section{Introduction \\ - Learning about dementia, stress in carers and understanding behaviours of the recipient of care. \\ Discussion \\ - Discussion of behaviours or situations that carers found difficult, incorporating behavioural management techniques, skills to take better care of themselves (including changing unhelpful thoughts), relaxation, increasing and assertive communication, promoting acceptance, sources of emotional support and positive reframing.}

\section{Future needs of the family member with dementia}

- Information about care and legal planning, specifically adapted to the UK. We gave the carers information leaflets about making common decisions as appropriate at an individual level.

\section{Planning pleasant activities}

- This used the idea that it is possible, beneficial and pleasurable to incorporate small pleasant activities into a caring day.

\section{Maintaining skills learnt over time}

- In the last session, the carer identified which techniques they found helpful and made a plan about what to continue for the future. Carers were given homework tasks to complete between sessions, including relaxation, identifying triggers and reactions to challenging behaviours and identifying and challenging negative thoughts. The therapist and the carer both had a manual, and the carer filled in and kept their own manual. Relaxation exercises used in sessions were recorded on a CD and given to the carers. We defined adherence to therapy on clinical grounds as participating in five or more sessions. therapies. The Prime Minister's Challenge on Dementia 2020 recommended that all family carers receive START or a similar intervention. ${ }^{8}$

Following completion of the START trial, we were funded by the Alzheimer's Society to disseminate the START intervention by cascading it through regional 'train-the-trainers' workshops. As in the original trial, we wanted START to be supervised by highly qualified clinical practitioners but delivered by those with less qualifications and experience. We trained a member of a clinical team. Interactive workshops were designed to provide the knowledge, materials and motivation to equip attendees to initiate the implementation of START into local practice. ${ }^{9}$ This approach was based on evidence that effective interventions to disseminate research findings into practice are usually multifaceted, comprising interactive teaching with feedback on practice and making plans to implement interventions. ${ }^{10}$ In contrast, passive dissemination of information may be ineffective as even when people agree with the material they often do not put it into practice. ${ }^{11}$ Although there are few evaluations of dissemination specifically within multidisciplinary teams, having an intervention promoted by one professional from the team seems to lead to its increased use within the team. ${ }^{7}$ In this study, we aimed to evaluate the extent to which our dissemination within train-the-trainer workshops enabled attendees to implement START in their clinical teams and what helped or hindered this over the year following course attendance.

\section{MATERIALS AND METHODS}

\section{Participants and procedures}

The Faculty for the Psychology of Older People in the British Psychological Society and Dementia UK for admiral nurses both advertised training opportunities through existing faculty and employee networks. They worked collaboratively with regional coordinators to maximise reach and use local free training venues. We trained clinical psychologists and admiral nurses to train graduate psychologists and other junior colleagues. Participants did not have to have any prior knowledge or interest in the START intervention and were given the manual to read in advance of the training session. Participants were given free training at 'train-the-trainers' workshops provided they committed to the evaluation.

\section{Workshop content}

PR, a clinical psychologist, involved in the development and trial of the START intervention facilitated 3-hour 'train-the-trainer' workshops (see table 1 for an overview of the training session). This began with interactive discussion of START, its use in practice and potential impact and how to train and supervise others to deliver the intervention. She summarised the evidence that individual, multicomponent interventions work better; that START can be delivered by supervised psychology graduates; the evidence for START being delivered in an 
Table 1 Overview of training sessions

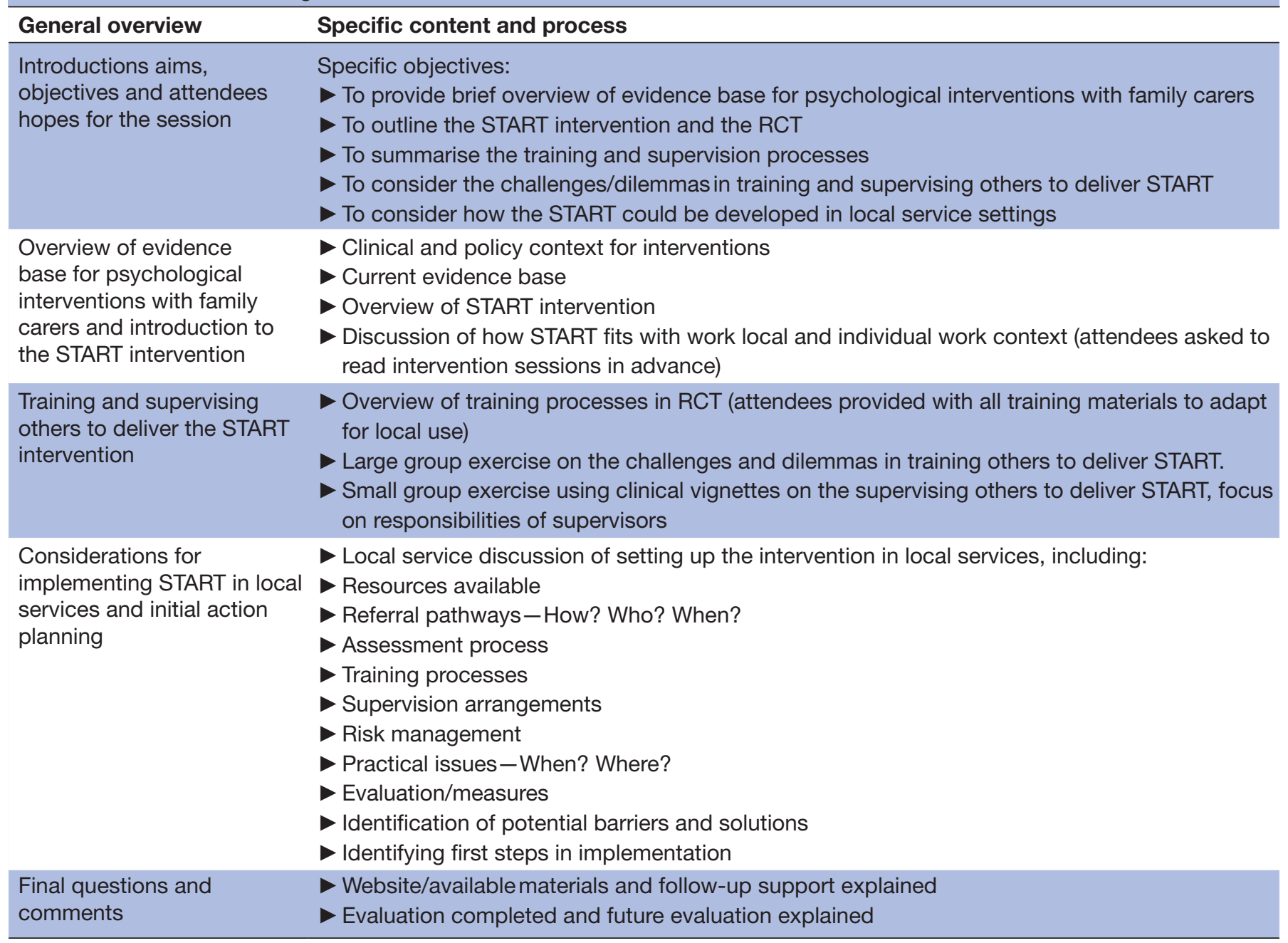

RCT, randomised controlled trial; START, STrAtegies for RelaTives.

individual therapy format and that it has been effective both as a preventative and as a therapeutic intervention. PR emphasised that START offered opportunities to improve existing care in a range of service contexts. Attendees were asked to think about next steps and what support or training they would need in order to take these steps in the feedback after the workshop. Attendees then discussed plans to promote and implement the intervention locally. PR emphasised that there was evidence-based trial evidence that START worked, which many other interventions do not have, and thus fidelity to the START manual was essential. Attendees were encouraged to implement START as it had been delivered in the original RCT, for example, as an individual rather than a groupbased intervention.

Members of the research team were available to answer any queries regarding START throughout the project, and we would respond to any questions with additional tailored advice, extra support materials and encourage links between those who attended the training experiencing similar challenges. We created a website providing free access to all study materials, including the START manuals, information sheets and necessary training materials to deliver and supervise START (http://www.ucl.ac. uk/psychiatry/start).

\section{Initial evaluation}

Attendees completed a short evaluation form on completion of the workshop regarding aspects of the training they found most and least useful, next steps they intend to take on returning to their service after completion of the workshop and what further training or support would they need in order to be able to take these steps.

\section{Evaluation survey at $\mathbf{6}$ and 12 months}

Attendees were emailed a short evaluation form on progress they had made with implementing START and what had helped or hindered this 6 and 12 months after the training. We asked how many family carers they had delivered START to personally, how many other professionals they had trained and their role and how many family carers had received START in their service in total. We asked who had delivered it. If we received no response 
within a few weeks, we sent two additional reminder emails unless the participant had moved.

\section{Interviews}

We invited Individuals who responded at the 1-year follow-up to carry out a telephone or face-to-face individual interview or attend a local focus group, whichever was most convenient for them. As others had not responded to multiple emails, we judged it unlikely they would take part in a face-to-face survey. We used a semistructured interview guide to explore if and how START was being delivered, the experience of the healthcare professionals locally when trying to implement START and what they felt would help with future implementation.

\section{Analysis}

\section{Qualitative data}

We audio recorded all interviews and transcribed them verbatim, removing all identifying information from transcripts prior to analysis. Qualitative data received from the email surveys and telephone interviews were managed, coded and analysed using the qualitative research software Nvivo 9. All data were thematically coded, generating a coding frame from initial interviews using a thematic content analytic approach. Disagreements between the researchers were resolved through discussion and a consensus was reached.

\section{Quantitative data}

All quantitative survey data were stored and analysed in Excel and basic descriptives generated.

\section{RESULTS}

One hundred and thirty-four clinical psychologists and 39 admiral nurses attended 14 training sessions between October 2014 and September 2015 in London, York, Doncaster, Cambridge, Edinburgh, Teeside, Port Talbot, Leicester and Birmingham.

\section{Workshop feedback}

All attendees completed a feedback evaluation immediately after completion of the training session. A summary of themes is presented in box 2. Attendees were generally very positive about the format and content of the training and suggested that longer training sessions may be helpful in the future. Many individuals agreed that their next steps would be to discuss the intervention with their clinical teams and managers and felt that continued support and contact with the research team would assist in doing this.

\section{Survey results}

In response to our initial email, 22 automatic responses were received saying that individuals no longer worked at that organisation. Of the remaining 151 original participants, 40/151 (26\%) people responded to the request for data across the 1-year follow-up period; $6(15 \%)$ were admiral nurses and $34(85 \%)$ were clinical psychologists.

\section{Box 2 Summary of workshop feedback}

What aspects of the training did you find most useful?

- Discussing implementation/vignette in local groups

- Discussing supervision dilemmas

- Hearing about the background to START and individuals' experiences of using it in clinical practice

What aspects of the training were least useful?

- Some of the local discussion about how to implement could have been done back among individuals' teams

What specific aspects of the training could be improved?

- Increase the length of the training, 3 hours not sufficient

- Hearing more from others who have implemented START

- Maybe have someone who currently delivers START or a carer who has received START present

\section{What will be your next steps in taking forward the START intervention in your service? \\ - Discuss with clinical leads and management \\ - Deliver START myself}

What further training/support would enable you to take these next steps?

- Support from and contact with the research team

- Examples of successful bids for funding to support this work

- Speak with other services using START

Of the 40 responding healthcare professionals who completed the survey, 16 have trained a total of 75 other healthcare professionals in using START and 11 have personally delivered START to 22 carers. Following training of colleagues, a total of 136 carers had received START across 11 different service areas (see table 2).

\section{Interviews}

Thirteen healthcare professionals took part in semistructured interviews/focus groups. We conducted 10 semistructured telephone interviews with clinical psychologists (one male, nine female) and one focus group with three admiral nurses (one female, two male). Seven of these healthcare professionals were currently delivering START in their services; the details of how are provided below.

\section{Facilitators of implementation (see box $\mathbf{3}$ for summary of themes)}

In areas where attendees were able to use START, there were a number of key themes identified by healthcare professionals that aided its implementation. These were as follows:

\section{Team sign up to START}

Raising awareness about the START intervention and its benefits among colleagues, including those who may not be directly involved in delivering it, for example, managers and referrers, was critical to the implementation process. 
Table 2 Numbers of colleagues trained and carers who have received START

\begin{tabular}{lccc}
\hline & Clinical psychologists & Admiral nurses & Total \\
\hline $\begin{array}{l}\text { Number of people who attended the training who } \\
\text { have personally delivered START to carers }\end{array}$ & 9 & 2 & 11 \\
$\begin{array}{l}\text { Number of colleagues in the service attendees have } \\
\text { trained }\end{array}$ & 75 & 0 & 75 \\
Number of carers who have received START & 130 & 6 & 136 \\
\hline
\end{tabular}

START, Strategies for Relatives.

We gave the team information and training, not training on how to do it but just information about what we were doing and why we were introducing this and the evidence base. And so, I think they were, they were fine with that as well. (Clinical psychologist)

Support from managers and other team members, referrers knowing what START involves, advocating for referrals in team meetings and feeding back 'success stories' to referrers all helped. (Clinical psychologist)

The motivation of the staff generally within the service to deliver START and awareness and recognition of the potential impact of START for carers encouraged implementation:

And I think just willingness really; you know people want to be doing everything. (Clinical psychologists)

I think within our service they've supported basically what we think is a good idea. (Clinical psychologist)

\section{Box 3 Summary of qualitative themes}

\section{Key facilitators of implementation}

Team sign-up to START

- Raising awareness of the need for the intervention

- Staff motivation and recognition of the potential impact of START

Familiarity of psychosocial interventions

- Existing skill set of colleagues

National and local policy drivers

- Carers support now on the national agenda

- Support from policy makers

Delivering a modified intervention

- Ability to tailor the intervention to their local arrangements

Access to START resources

- Free availability of all the intervention resources

\section{Barriers to implementing the START intervention}

Lack of time and resources

- Times required to set-up the intervention locally

- Lack of junior colleagues to deliver the intervention

- Service cuts there struggle to provide 'extra' interventions

Lack of buy-in and support from colleagues and managers

- Lack of managerial support for a 'new' intervention

- Colleagues not feeling they have the skills to deliver or supervise this type of intervention

Lack of fit with current service context or professional approach

- Complexity of clients on caseload

- Lack of experience delivering such interventions
Familiarity with psychosocial interventions

Within the psychology services, many of the respondents referred to the existing skill set of their colleagues and their competency in delivering these types of psychological interventions as aiding the implementation of START:

Certain parts of it [START] that are very kind of familiar to them in the work that we do...I we already had a group of people in our team who are quite skilled and quite used to going out to see people one on one and talking through sometimes quite emotive topics, building links and they didn't need that much support really, in terms of implementing it. (Psychologist)

\section{National and local policy drivers}

There were local policy drivers identified that aided the implementation of START locally. Providing support interventions for carers of people with dementia is on the national agenda across the UK, with START being a recommended intervention. Healthcare professionals spoke of the commissioning pressures to show how their psychology services were exploring how to provide such support:

In terms of commissioning, you need to be seen to be doing these things. So I think it works in START's favour if you know, if it's got good evidence, then people are going to want to do it. (Clinical psychologist)

I think there's huge focus now on supporting carers and I think that's probably a reflection of me having more one-toone referrals as well. And more acknowledgment really of the emotional, psychological needs of carers rather than just the information given. Yes, definitely within my service anyway, there's a huge focus now for supporting carers. (Clinical psychologist)

With carers support on the agenda, in some areas, healthcare professionals spoke of increased funding to provide this locally, with START being one of the ways of doing so:

There is direct funding within the council from a dementia stream, for us to be involved in some dementia work locally. (Psychologist)

In one site, having the support from policy makers working on the dementia agenda within the local council fostering inter-agency working with the third sector 
facilitated referral of suitable carers for the delivery of START to carers:

She [policy officer] already had links with [the third sector] so she had two points of contact there, who were then able to drive referrals for her. (Psychologist)

\section{Delivering a modified intervention}

Healthcare professionals were trained to deliver and supervise START in the format used in the original trial. A number of services have made the decision to deliver a variant of START, for example, as a group intervention:

I'm the only psychologist obviously covering quite a large area, I don't have the resource to work one-to-one. (Clinical psychologist)

Despite the lack of evidence that START works this way, these sites felt this was the most effective way to see as many carers as possible and they had support from their services to use this approach. Similarly, in relation to the format of supervision necessary for colleagues delivering START, individuals were adopting less formal arrangements in terms of frequency and this was mentioned as helpful:

It has been relatively ad hoc... we're actually sitting next to each other all the time, it's really if they've come back, and anything particular that has been difficult, we talk about. (Psychologist)

\section{Access to START resources}

The free availability of the START resources via both the website and the research team was helpful to healthcare professionals and encouraged implementation. Additionally, email reminders and regular contact from the research team kept the START intervention in people's minds and on their agenda:

I think just the fact it's been made very accessible, because I think often with interventions, there's lots and lots of hoops to jump through, and just the fact that all the materials are there, it's really handy really, so that's definitely been positive. (Clinical psychologist)

Just knowing that there's a contact, so yourself or others that we can contact for information. (Clinical psychologist)

\section{Barriers to implementing the START intervention}

On the basis of the responses received, in three of the geographical areas where individuals attended the trainthe-trainers sessions and subsequently trained their colleagues, no carers had received START. Reasons for this were as follows: a lack of appropriate referrals, that is, START was not thought by other professionals to be suitable for their current caseload; other demands on the service and START not being seen as a priority; lack of assistant psychologists to deliver START; the person who attended the START training went on maternity leave and the amount of time needed to set-up the intervention within the service.
Lack of time and resources

Many respondents spoke of the amount of time required to set up a new intervention within a service and 6 months after the training session, very few had made any progress with implementation. This was because of the time needed to plan and organise how START would be delivered within the service, including getting colleagues on board, setting up the referral process and agreeing who would be responsible for delivery:

As this is a 'new' thing (manualised intervention) has taken a long time to get everyone on board, work through all the practical issues of delivery. (Clinical psychologist)

It's just taken a few months to get everything up and running. (Clinical psychologist)

So this is a new thing really that we've had to, kind of, it's taken us months just to get people on board and share our ideas really and organise, even practical things, where it'll take place, so yes it has taken us a long time to get this far really. (Clinical psychologist)

At the 1-year follow-up, this was beginning to change, and START was being delivered in some of these areas:

It was maybe about a year, a year and a half before it ever actually started to be delivered. (Psychologist)

It took so long just to get it off the ground... over a year now. (Clinical psychologist)

An initial consideration for those wishing to implement START locally was who was available within their service to deliver the intervention. Several psychologists, especially those outside of London, reported that there were too few psychologists, usually just one, covering a large geographical area with a very full caseload and so they could not supervise assistant psychologists. In addition, many individuals lacked psychology assistants or alternative junior colleagues within their service:

No, in fact it's just one and a half psychologists for the whole service. We used to be seven. (Clinical psychologist)

We need more staffing resource. At the moment staff are struggling to do the basics and the focus in on assessment and diagnosis, so there is a difficulty in introducing new interventions or even in being able to appoint, train and supervise volunteers. (Clinical psychologist)

Due to current and planned resource cuts within services, a number of participants felt that there was a lack of financial resource available to deliver START and that other demand within the service were currently taking priority:

I don't know whether they (the service) would be supportive in terms of ring-fencing time to organise it which is what makes me slightly hesitant about doing this, if you see what I mean? (Clinical psychologist)

Not currently seen as a priority given service target pressures and service reorganisations. (Clinical psychologist) 
Healthcare professionals spoke about the challenge of balancing this lack of resources with the demand for the START intervention in terms of numbers of carers needing to be seen and the time this would take:

\section{So I think it's difficult because that would mean extra time} that I don't have at the moment. (Clinical psychologist)

In the memory service, demand outstrips supply, engaging carer's takes a lot of time, as does managing risk issues which arise. (Clinical psychologist)

They also reported that they were struggling to cover all the content of a session within 1 hour and several felt that asking for eight sessions of their time to deliver the intervention was not going to be possible:

I think that's going to be the crux, you know is it... do people... can they justify eight contacts basically? (Clinical psychologist)

My manager did think that was an idea... but when she realised it was eight week sessions, not one, she said, no... they're struggling to meet the demands of the assessments they have to make they've got payment by results, so they're struggling already. So to give them something else to do, although they recognise it is a good thing to do, unfortunately it comes down to time and resource. (Admiral nurse)

Professionals also discussed the perceived limitations of delivering a one-to-one intervention and that other interventions delivered in a group were seen as more cost and time effective (even if lacking evidence of clinical or cost-effectiveness):

I am involved in interventions for carers and have found delivering these through groups more time efficient. (Clinical psychologist)

\section{Lack of buy-in and support from colleagues and managers}

Many healthcare professionals talked about how they had to justify that the START intervention comes within their role and is a worthwhile use of their time. In order to do so, it required someone to 'champion' the use of START. Absence of support from a managerial perspective also hindered the implementation of START and priority was not being given to support this process:

\section{I battled for it. (Clinical psychologists)}

I have also not been successful in finding a manager who is interested in taking this up or exploring it further. I think that there is a lack of identified need in this area which doesn't help. (Clinical psychologist)

Some clinical psychologists who had tried to implement START in their local services felt other team members and management were reluctant to adopt START. The potential reasons included the following: a lack of interest from colleagues to deliver and provide psychological approaches to support carers, colleagues not feeling skilled to deliver START or the assumption that they did not have the necessary time or resources to provide START:

I didn't have a very good response from CPNs [Community Psychiatric Nurses], unfortunately. Some of them don't feel as skilled in that. Some people talked about having limited time, other people not really being interested in psychological approaches. I think a lot of people probably don't have the confidence, they have the skills I think but maybe not the confidence to deliver it. (Clinical psychologist)

Lack of fit with current service context or professional approach Several healthcare professionals felt that they were supporting carers with very complex needs, especially those based in Community Mental Health Teams (CMHTs), and that START was not appropriate at that time or did not fit with their professional identity or the ethos of their clinical services. Admiral nurses also felt that they were very skilled in delivering carers support and were already providing much of the content of the START intervention without using its structured approach:

Admiral Nurse model responds to the highest expressed need of the carer and then moves on to other areas, so this very structured programme may not fit with that. (Admiral nurse)

For carers that would need this level of input as a team we provide the Educational programme for 5 weeks for carers and a lot of the information is covered within the course... In relation to the carers they tend to react to a situation as it occurs therefore even when we have covered the educational side within our Positive Steps Course we are often back involved as a major caring transition occurs within their role and the focus therefore will be on the emotional support surrounding this particular identified need. (Admiral nurse)

When discussing staff skills, clinical psychologists felt they had the ability to supervise junior members of staff and that this was a usual part of their role. In contrast, admiral nurse participants felt that they have very little supervision experience, and this was not part of their current role and did not fit in with their service structure:

Not all admiral nurses have got experience in giving supervision. I mean, some have come through, and some have been ward managers and team leaders and are used to that environment of giving supervision. But that's something that might need some skills development if they're going to do that. (Admiral nurse)

\section{DISCUSSION}

We were able to attract clinical psychologists and admiral nurses professionals from across the country to our workshops about the START intervention and they evaluated these positively. We delivered 14 interactive training sessions, for each of which almost 10 family carers received the intervention in the year after training. Despite the cheapness of our project, the dissemination 
may not have been good value. It was certainly not sufficient, as relatively few people received the intervention and the immediate benefit was relatively low. We did not record delivery after this but are aware that the services we worked with are continuing to deliver START and embed it in their services as they frequently contact us in relation to their ongoing work. Developing and evaluating new interventions is expensive, but many evidence-based interventions are either not implemented in real-world practice or implemented after a long time. This small, relatively inexpensive dissemination study began this process by providing the information, support and materials to clinicians that are necessary but not sufficient for implementation in local services. We also considered what would help further implementation to inform future work.

Those who attended workshops are likely to have been more open minded and amenable to change. We found that even for those workshop attendees who were highly motivated, it took considerable time to implement the intervention. Some clinical psychologists have said that they wish to implement START by training others but are constrained by the resources available and multiple competing demands on their time. Although training assistant practitioners to deliver the intervention could theoretically be a cost-effective way of bringing psychological treatments to more family carers, in practice many clinical psychologists did not have more junior colleagues they could train, so they were unable to change a situation where only a few family carers with very high needs were seen by more expensive, fully trained psychologists. Staff interviewed also reported feeling that the START intervention was more time and resource intensive than usual service provision, for example, delivering a psycho-educational group course, despite evidence suggesting that these interventions are ineffective for carer mood. ${ }^{12} 13$ In many cases, the lesser resource implications and possibly motivation needed to continue with interventions currently being delivered appeared to mitigate against introduction of START, although these interventions had a lesser evidence base for reducing carer psychological morbidity. This disconnect between research evidence and practice has been highlighted in other areas of dementia research, ${ }^{14}$ and there is perhaps a need to evidence how START can be effective in a diverse range of service settings to reflect the contexts where family carers are most likely to access psychological support.

It was interesting to reflect on the different experiences of admiral nurses compared with clinical psychologists in implementing START. It was evident from our findings that the intervention fitted less well with their ethos, current models of service delivery and professional identity. Although START trials included an admiral nurse service, there was a reluctance to accept that the evidence base for it applied to the context of their work. Admiral nurses often work autonomously in senior roles and do not usually supervise others. Family carers value the service they give, which is perhaps more defined by experience than the current evidence base; we think all services would benefit from incorporating current best evidence into their work and will continue to explore ways to encourage all services to implement it.

We did not explicitly ask attendees whether they had access to a pool of junior colleagues to train when they signed up for the training, as we prioritised reach and dissemination of START over targeted implementation. However, attendees were informed that the intervention was intended to be delivered by junior colleagues and the attendees were expected be in a position to train and develop START locally. Those participants who worked in memory clinics and CMHTs had some junior colleagues but most admiral nurses did not.

We experienced many difficulties in finding out what is happening at local sites, if they are using START and how. Our low response rate means we have much less information than planned, and we think that it is likely that those who did not respond had not implemented the intervention, although many had moved services, and it is possible but likely to be relatively rare that they took their knowledge and skills to their new services. This may be due to the fact that we were asking them to complete an email survey. We invited attendees to get in touch with the research team if they had any queries or issues regarding START, but many did not make any contact following the training. We know from the 22 out of office responses to emails that there is currently a high level of movement within services. Our qualitative evaluation was only from those who had answered to begin with and, therefore, is likely to include those who were more interested or successful. The complex landscape of service delivery reinforces the need for multifaceted, targeted, implementation strategies. One of the services that offered START to the most carers was one where local commissioners had been approached and allocated specific resource to set up and deliver the intervention across the borough.

We have made all of our training materials and the START manuals freely available to widen potential access to the intervention for family carers. This means that we have no control over how the intervention is delivered and we had no way of monitoring fidelity although we emphasised its importance. From the feedback we received, a number of sites are using a different format and process to deliver the intervention than that tested in our RCT, for example, delivering in groups or delivering to professional carers. We do not have data on the effectiveness of the intervention when delivered in this way, and we have no measure of fidelity or adherence to the original intervention. This raises an interesting question about the implications this may have on the effectiveness of the intervention if not delivered in the intended way with a trade-off between flexibility and treatment fidelity, which has been noted in other areas of dementia care. ${ }^{15}$

Disseminating START in this 'train-the-trainer' way is the beginning of the implementation process, but by itself we have found that it is not enough to ensure 
implementation into practice. We disseminated START broadly and provided individuals with the materials they need to train others and to set up the intervention locally. This motivated a small group of individuals whom one could characterise as 'early adopters' to address barriers and implement START locally for at least some patients. A range of top-down and bottom-up approaches are needed to implement more broadly. As sites are deviating from the original study design as to how and by whom START is delivered, exploring further who are the right people to be delivering START locally, future implementation should evaluate fidelity or in its absence outcome. Our in-depth case studies of 'real-world' implementation in a variety of settings may help other areas implement START. This work would also allow us to look further into the role of specialist professionals such as admiral nurses as their model of delivery is very different from clinical psychology and they tend not to work in a team structure with junior colleagues. Interventions are more likely to be implemented when there are resources available, the staff see there is a benefit and they feel it provides value. ${ }^{16}$ Our next step is to take a more tailored approach to START and to lay the foundation to deliver it in other contexts, for example, outside the health system or to different ethnic groups.

Acknowledgements We would like to thank the professionals who took part in our interviews.

Contributors GL was chief investigator for the project grant. PR and CC were grant co-investigators. PR delivered all the START training to healthcare professionals, and $\mathrm{KL}$ organised the training sessions and completed the qualitative interviews and data collection. $\mathrm{KL}, \mathrm{PR}$ and $\mathrm{GL}$ analysed the qualitative interview data. $\mathrm{KL}$ wrote the first draft of the paper. All authors (GL, PR, CC and KL) revised the paper critically.

Funding This work was supported by the Alzheimer's Society, grant number 217 AS_DG_13_0002.

Competing interests $\mathrm{PR}, \mathrm{GL}$ and $\mathrm{CC}$ developed the original intervention and were co-applicants in the RCT.

Provenance and peer review Not commissioned; externally peer reviewed.

Data sharing statement There are no unpublished data from the study.

Open Access This is an Open Access article distributed in accordance with the Creative Commons Attribution Non Commercial (CC BY-NC 4.0) license, which permits others to distribute, remix, adapt, build upon this work non-commercially, and license their derivative works on different terms, provided the original work is properly cited and the use is non-commercial. See: http://creativecommons.org/ licenses/by-nc/4.0/ (c) Article author(s) (or their employer(s) unless otherwise stated in the text of the article) 2017. All rights reserved. No commercial use is permitted unless otherwise expressly granted.

\section{REFERENCES}

1. Prince M, Knapp M, Guerchet M, et al. Dementia UK: update. 2014. https://www.alzheimers.org.uk/site/scripts/download_info.php? filelD=2323.

2. Livingston G, Barber J, Rapaport P, et al. Clinical effectiveness of a manual based coping strategy programme (START, STrAtegies for RelaTives) in promoting the mental health of carers of family members with dementia: pragmatic randomised controlled trial. BMJ 2013;347:f6276.

3. Brodaty H, Donkin M. Family caregivers of people with dementia. Dialogues Clin Neurosci 2009;11:217-28.

4. Livingston G, Barber J, Rapaport P, et al. Long-term clinical and cost-effectiveness of psychological intervention for family carers of people with dementia: a single-blind, randomised, controlled trial. Lancet Psychiatry 2014;1:539-48.

5. Knapp M, King D, Romeo R, et al. Cost effectiveness of a manual based coping strategy programme in promoting the mental health of family carers of people with dementia (the START (STrAtegies for RelaTives) study): a pragmatic randomised controlled trial. BMJ 2013;347:f6342.

6. Teri L, McKenzie G, Logsdon RG, et al. Translation of two evidencebased programs for training families to improve care of persons with dementia. Gerontologist 2012;52:452-9.

7. Döpp CM, Graff MJ, Rikkert MG, et al. Determinants for the effectiveness of implementing an occupational therapy intervention in routine dementia care. Implement Sci 2013;8:1-16.

8. Prime Minister's Challenge on dementia 2020. 2015.

9. Kristensen N, Nymann C, Konradsen H. Implementing research results in clinical practice- the experiences of healthcare professionals. BMC Health Serv Res 2016;16:1-10.

10. Grol R, Grimshaw J. From best evidence to best practice: effective implementation of change in patients' care. Lancet 2003;362:1225-30.

11. Gvozdenović E, Allaart CF, van der Heijde D, et al. When rheumatologists report that they agree with a guideline, does this mean that they practise the guideline in clinical practice? results of the International Recommendation Implementation Study (IRIS). RMD Open 2016;2:e000221.

12. Selwood A, Johnston K, Katona C, et al. Systematic review of the effect of psychological interventions on family caregivers of people with dementia. J Affect Disord 2007;101:75-89.

13. Corbett A, Stevens J, Aarsland D, et al. Systematic review of services providing information and/or advice to people with dementia and/or their caregivers. Int J Geriatr Psychiatry 2012;27:628-36.

14. Fossey J, Masson S, Stafford J, et al. The disconnect between evidence and practice: a systematic review of person-centred interventions and training manuals for care home staff working with people with dementia. Int J Geriatr Psychiatry 2014;29:797-807.

15. Jutkowitz E, Brasure M, Fuchs E, et al. Care-delivery interventions to manage agitation and aggression in dementia nursing home and assisted living residents: a systematic review and meta-analysis. $J$ Am Geriatr Soc 2016;64:477-88.

16. Minard LV, Deal H, Harrison ME, et al. Pharmacists' perceptions of the barriers and facilitators to the implementation of clinical pharmacy key performance indicators. PLoS One 2016;11:e0152903. 